\title{
IKKE cooperates with either MEK or non-canonical NF-kB driving growth of triple-negative breast cancer cells in different contexts
}

\author{
Carrie D. House, Valentina Grajales, Michelle Ozaki, Elizabeth Jordan, Helmae Wubneh, Danielle C. Kimble, \\ Jana M. James, Marianne K. Kim and Christina M. Annunziata* (D)
}

\begin{abstract}
Background: Metastatic breast cancer carries a poor prognosis despite the success of newly targeted therapies. Treatment options remain especially limited for the subtype of triple negative breast cancer (TNBC). Several signaling pathways, including NF-KB, are altered in TNBC, and the complexity of this disease implies multi-faceted

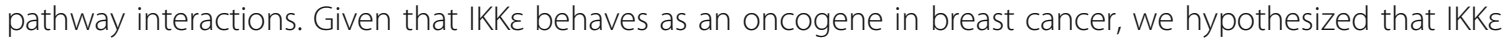
regulates NF-KB signaling to control diverse oncogenic functions in TNBC.

Methods: Vector expression and RNA interference were used to investigate the functional role of IKKE in triple-negative breast cancer cells. Viability, protein expression, NF-kB binding activity, invasion, anoikis, and spheroid formation were examined in cells expressing high or low levels of IKKE, in conjunction with p52 RNA interference or MEK inhibition.

Results: This study found that non-canonical NF-KB p52 levels are inversely proportional to IKKE, and growth of TNBC cells in anchorage supportive, high-attachment conditions requires IKKE and activated MEK. Growth of these cells in anchorage resistant conditions requires IKKE and activated MEK or p52. In this model, IKKE and MEK cooperate to support overall viability whereas the p52 transcription factor is only required for viability in low attachment conditions,

underscoring the contrasting roles of these proteins.

Conclusions: This study illustrates the diverse functions of IKKE in TNBC and highlights the adaptability of NF-KB signaling in maintaining cancer cell survival under different growth conditions. A better understanding of the diversity of NF-KB signaling may ultimately improve the development of novel therapeutic regimens for TNBC.
\end{abstract}

Keywords: NF-kappaB, Triple negative breast cancer, IKK-epsilon, Non-canonical signaling, Anoikis

\section{Background}

Breast cancer results in approximately 40,000 deaths per year in the United States [1]. Despite new therapies designed to target different subtypes of breast cancer, there remains a poor prognosis for metastatic disease. A further understanding of the molecular mechanisms required for tumor cell survival during the process of metastasis and relapse will aid in the development of more targeted therapies for breast cancer, especially in the case of the triple-negative breast cancer (TNBC) subtype for which treatment options are limited.

\footnotetext{
* Correspondence: annunzic@mail.nih.gov

Women's Malignancies Branch, National Cancer Institute, Bethesda, MD, USA
}

TNBC is a heterogeneous disease characterized by an absence of well-defined targets and a poor five-year survival rate for metastatic disease. Gene expression and ontology studies have further characterized TNBC into as many as six molecular subtypes that include basal-like and non-basal like tumors $[2,3]$. Recognition of individual subtypes in patients will likely lead to better therapeutic strategies; the complexity associated with TNBC, however, implies an increased level of pathway cross-talk and compensatory mechanisms [4]. Signaling pathways altered in TNBC progression include p53, PI3K, MEK, and NF-kB, among others [4-10] with new clinical trials underway using novel combinations of different pathway inhibitors [4]. 
NF- $\kappa \mathrm{B}$ is a signaling pathway important for controlling immune response, stress response, cell survival, proliferation, differentiation, and apoptosis [11-15]. Activation of NF- $\kappa \mathrm{B}$ is mediated by the I $\mathrm{B}$ k kinases $\mathrm{IKK} \alpha, \mathrm{IKK} \beta$, and $\mathrm{IKK} \varepsilon$, resulting in nuclear localization of NF- $\mathrm{kB}$ transcription factors (c-Rel, RelA, RelB, p105/50 and p100/52) and subsequent transcriptional activation [16]. There are both canonical and non-canonical pathways operating within the NF- $\mathrm{B}$ network, allowing tight regulation of various biological functions [17]. Activation of NF-kB can occur through multiple stimuli, and this pathway interacts with other prominent signaling pathways, although the molecular mechanisms contributing to cancer progression remain unclear [16, 18-21]. Our laboratory has previously characterized NF- $\mathrm{kB}$ activation downstream of IKK $\beta$ and IKK $\varepsilon$ in ovarian cancer, and several studies support that NF- $\mathrm{kB}$ is an important contributor to cancer progression and chemoresistance [17, 22-24]. Expression analysis of TNBC tissue with adjacent normal breast tissue suggests that NF- $\mathrm{B}$ is a key regulator of the molecular TNBC phenotype [5].

The kinase IKKe (encoded by IKBKE gene) has been shown to be an oncogene in breast $[20,25,26]$ and ovarian [24] cancers. Silencing of IKBKE reduced proliferation, clonogenicity, migration and invasion of breast cancer cells $[20,27]$. IKK $\varepsilon$, in cooperation with MEK, can function as a transforming kinase in human mammary epithelial cells [20]. Most studies have focused on IKKe function in the luminal subtype, whereas the role of this kinase in the more aggressive basal subtype has only recently been explored. In that setting, IKKe in combination with $\mathrm{Jak} /$ Stat signaling may promote cytokine activation that induces tumorigenesis in an immune-activated subtype of TNBC. Although $\mathrm{IKK} \varepsilon$ is known to phosphorylate one of two acceptor sites of $\mathrm{I} \kappa \mathrm{B} \alpha$, its role in NF- $\kappa \mathrm{B}$ activation remains unclear. Given the broad activity of NF- $\mathrm{kB}$, our work presented here seeks to clarify whether this kinase supports canonical or non-canonical signaling and, furthermore, what oncogenic features depend on this signaling circuit.

\section{Methods}

\section{Cell lines and culture conditions}

Breast cancer cell lines MDA MB 231 (cat. No. HTB-26) [claudin-low TNBC], MDA MB 453 (cat. No. HTB-131) [HER2 (ER-,PR-, HER2+)], MDA MB 468 (cat. No. HTB-132) [basal TNBC], HCC-38 (CRL-2314) [claudin-low TNBC], BT-549 (cat. No. HTB-122) [basal TNBC], and BT-474 (cat. No. HTB-20) [luminal B (ER-, PR+,HER2+] were purchased from American Type Culture Collection (ATCC, Manassas, VA). Unless otherwise noted, all breast cancer cell lines were cultured in RPMI 1640 (Gibco, Thermo Fisher, Grand Island, NY) containing 10\% FBS (Gemini, West Sacramento, CA) and 1\% penicillin/streptomycin (Gibco, Thermo Fisher, Grand Island, NY) and maintained at $37^{\circ} \mathrm{C}$ in a $5 \% \mathrm{CO}_{2}$ atmosphere.

\section{Expression and shRNA constructs}

pBabeNeo (plasmid \#1767) and pBabe-Neo-Flag-IKBKE (plasmid \#15265) were purchased from Addgene. Transduced cells were cultured in the presence of $200 \mu \mathrm{g} / \mathrm{ml}$ neomycin for 7 days. Use of IKBKE short hairpin (shRNA) constructs has been previously described [24]. Two rounds of viral supernatants were applied to breast cancer cell lines over the course of $48 \mathrm{~h}$, followed by incubation with growth medium for $24 \mathrm{~h}$ and selection with $2 \mu \mathrm{g} / \mathrm{mL}$ puromycin for 7 days. Selected transduced cells were used for all assays. Sequences of shRNA constructs: non-targeting control (shNeg):

\section{forward GATCCCCTCTCAACCCTTTAAATCT GATTCAAGAGATCAGATTTAAAGGGTTGAG AGTTTTT, reverse AGCTAAAAACTCTCAACCC TTTAAATCTGATCTCTTGAATCAGATTTAA AGGGTTGAGAGGG.}

shIKKe 1:

forward GATCCCGAGAAGTTCGTCTCGGTCTATTT CAAGAGAATAGACCGAGACGAACTTCTCTTTTT, reverse AGCTAAAAAGAGAAGTTCGTCTCGGTCTA TTCTCTTGAAATAGACCGAGACGAACTTCTCGG.

$\operatorname{shIKK\varepsilon ~2:~}$

forward GATCCCGAGAGCCTCCTGTTCTTT CTATTCAAGAGATAGAAAGAACAGGAGGCT CTCTTTTT. reverse AGCTAAAAAGAGAGCCTCCTGTTCTTTCT ATCTCTTGAATAGAAAGAACAGGAGGCTCTCGG.

\section{siRNA transfections}

Cells were cultured for $24 \mathrm{~h}$ to $50 \%$ confluence before transfection with Dharmacon On-Targetplus SMARTpool short interfering (siRNA) duplexes (NF-kB2, cat. No. L-003918-00; non-targeting control, cat. No. D-001810-10; IKBKE, cat. No. L-003723-00) according to manufacturer's instructions (GE Dharmacon, Lafayette, CO). Briefly, cells were transfected with Dharmafect 1 transfection reagent (GE Dharmacon, Lafayette, CO) and individual siRNAs at a final concentration of $1 \% \mathrm{v} / \mathrm{v}$ and $25 \mathrm{nM}$, respectively. Cells were maintained in the presence of transfection reagent under normal culture conditions for $24 \mathrm{~h}$ before being used in assays.

RNA extraction and quantitative real-time PCR (qRT-PCR) Total RNA was extracted using the RNeasy Mini Kit (Qiagen) per manufacturer's instructions and treated with DNAse. Final RNA concentration was determined using a NanoDrop spectrophotometer. RNA was reverse transcribed using Taqman reagents (Applied Biosystems) 
and gene expression was measured using Taqman probes on a ViiA7 Real-time PCR machine (Applied Biosystems). GAPDH was used as a control and quantitation of gene expression was accomplished using comparative threshold cycle $\Delta \Delta \mathrm{C}_{\mathrm{T}}$. Primers were purchased from Applied Biosystems (p52 cat. No. Hs01028901_g1, CXCL1 cat. No. Hs00236937_m1, CD44 cat. No. Hs01075861_m1, and GAPDH cat number: 4325792.

\section{Western blot}

Whole cell protein was extracted from breast cancer cell lines using standard methods with NP-40 lysis buffer. Protein concentrations were determined using BCA Protein Assay Kit (Pierce, Thermo Scientific, Rockford, IL). SDS-PAGE was performed using the NuPage system (Invitrogen) and Luminata HRP Chemiluminescent Detection Reagents (Millipore, Temecula, CA). Antibodies were purchased from Sigma (IKKع, cat. No. I4907),

Abcam (IKK $\beta$, cat. No. ab32135), Millipore (GAPDH, cat. No. MAB374; p100/52 cat. No. 05-361), Santa Cruz (p65, cat. No. sc-372), and Cell Signaling (IKK $\alpha$, cat. No. 2682; pERK1/2, cat. No. 4377; Erk1/2, cat. No. 9102; phospho-p-65 (Ser536), cat. No. 3033).

\section{Chromatin immunoprecipitation-qPCR (ChIP-qPCR) assay}

The SimpleChIP Enzymatic Chromatin IP Kit (magnetic beads) was purchased from Cell Signaling Technology (Danvers, MA). Assays were performed according to the manufacturer's instructions. The antibody for p52 was purchased from Santa Cruz Biotechnology (cat. No. sc-7386 X). Promo was used to evaluate DNA sequences for transcription factor binding sites (http://alggen.lsi.upc.es/cgi-bin/promo_v3/ promo/promoinit.cgi?dirDB=TF_8.3). The first 5000 bases upstream of the transcription start site were screened for binding motifs that correspond to NF-kB consensus binding sequence. The quantification of transcription factor binding to target genes was calculated by measuring the ratio of ChIP-to-Input and normal rabbit IgG antibody served as a negative control. Primer sequences for NF-kB binding sites on $C X C L 1$ promoter: - 2.5 kb site: forward GATTTCCAGGCTCAAGGATGTA, reverse TCATTCAGTCTTCCAAACAAGC; - 02. Kb site: forward ATCCCAGAGTCTCAGAGTCCAC, reverse $A A A$ TTCCCGGAGTTCCAGAT.

\section{Co-immunoprecipitation assay}

Immunoprecipitation was performed on MDA MB 468 cells using the Abcam immunoprecipitation kit (cat. No. ab206996), according to manufacturer's instructions. Briefly, non-denaturing lysis buffer was used to collect 300 $\mu \mathrm{g}$ of cell lysate was incubated overnight with $3 \mu \mathrm{g} / \mathrm{ml}$ of either control rabbit IgG (Santa Cruz, cat. No. sc-2027) or IKK $\varepsilon$ rabbit polyclonal antibody (Abcam, cat. No. ab7891).
Antibody bound proteins were captured using protein A/ $\mathrm{G}$ sepharose beads, eluted, and analyzed via western blot. Antibodies used for western blot detection were purchased from Sigma (IKKع, cat. No. I4907), Santa Cruz (IKK $\alpha$, cat. No. sc-7606 and NIK cat. No. sc-8417).

\section{Viability assay}

MEK inhibitor, AZD6244, and a non-selective inhibitor of Ser/Thr kinases, BX795, that inhibits IKK $\varepsilon$, among others were purchased from Selleck Chemicals (Houston, TX). The IKK $\beta$ inhibitor IKK-2 inhibitor IV from Calbiochem (San Diego, CA). The breast cancer cell growth was assessed using XTT as described [28]. Cells were seeded in 96-well plates at a density of 2000 cells/50 $\mu \mathrm{l} /$ well. Plates were incubated for up to 9 days with medium and/or drug replenished every 3-4 days. Growth was assessed by incubating cultures with XTT for $3 \mathrm{~h}$ and absorbance read in a Tecan plate reader (Research Triangle Park, NC). Cell density in experimental wells was expressed as percent control. Experiments included triplicate samples and were repeated at least three times. IC50 values were calculated using CalcuSyn software (Paramus, NJ) and compared with publicly available database (www.cancerrxgene.org).

\section{NF-kB immunoassay}

Nuclear lysates were extracted using a Nuclear Extraction Kit according to manufacturer's instructions (Active Motif, Carlsbad, CA). Protein concentrations were determined using BCA Protein Assay Kit (Pierce, Thermo Scientific, Rockford, IL). NF- $\mathrm{kB}$ transcription factor binding was assessed using the TransAM NFКB Family ELISA Kit according to manufacturer's instructions (Active Motif, Carlsbad, CA). $10 \mu \mathrm{g}$ of nuclear extracts/20 $\mu \mathrm{l} /$ well were analyzed in triplicate and repeated at least three times.

\section{Invasion assay}

Invasion potential of breast cancer cells was assessed using Cultrex 96-well BME Cell Invasion Assays (Trevigen, Gaithersburg, MD) according to manufacturer's specifications. Briefly, $5 \times 10^{4}$ cells suspended in serum-free RPMI were plated in BME coated chambers, and allowed to migrate for $48 \mathrm{~h}$ using RPMI containing 10\% FBS as a chemoattractant. Cells that migrated through BME chambers were stained with calcein, solubilized, and numbers assessed by measuring fluorescence in a Tecan fluorimeter (Tecan, Research Triangle Park, NC). Migrated cell numbers in triplicate samples were reported as percent control.

\section{Anoikis assay}

Anchorage-independent, low-attachment (LA), growth was evaluated using the CytoSelect 96-Well Anoikis Assay according to manufacturer's protocol (Cell Biolabs, Inc., San Diego, CA). Briefly, $1 \times 10^{4}$ cells/100 $\mu \mathrm{l} /$ well were plated in quadruplicate on a 96-well anchorage resistant plate and in a 
companion standard culture plate with cell high attachment capability (HA) plate and cultured for $48 \mathrm{~h}$. Anoikis was assessed by dual staining with calcein AM and ethidium homodimer followed by measurement of fluorescence in a Tecan fluorimeter (Tecan, Research Triangle Park, NC). Experiments were repeated at least three times. Fluorescence intensity was expressed relative to control treatment.

\section{Spheroid formation assay}

To generate breast cancer spheroids, 500 cells/well were cultured in serum free media containing EGF $(20 \mathrm{ng} / \mathrm{ml})$ and FGF (10 ng/ml) (Sigma-Aldrich, St. Louis, MO) in ultra-low attachment 96 well plates (Corning, Corning, $\mathrm{NY}$ ). After $96 \mathrm{~h}$, spheroids were imaged using AxioVision Rel. 4.8 through an Axio Observer A1 Inverted Microscope (Zeiss) and analyzed using ImageJ Software 1.48v [29]. Spheroids with diameter $\geq 50 \mu \mathrm{m}$ were quantified. Spheroid efficiency was calculated using the formula (spheroids $\geq 50 \mu \mathrm{m} /$ cells per well).

\section{Statistical analysis}

All statistical analysis was performed using Prism (GraphPad) using data acquired from at least three biological replicates. $P<0.05$ was considered statistically significant. $P$ values were calculated as described in figure legends. Error bars represent standard error of the mean.

\section{Results}

The protein levels of IKK $\varepsilon$ in breast cancer cell lines of different molecular subtypes were surveyed. Consistent with previous reports [20], IKK $\varepsilon$ expression was variable and independent of basal or luminal status (Fig. 1a). Since $\mathrm{IKK} \varepsilon$ has been shown to activate the NF- $\mathrm{kB}$ pathway and cooperate with MEK to induce transformation in breast cells, the sensitivity of the breast cancer cells to inhibiting $\mathrm{IKK} \varepsilon$, IKK $\beta$ or MEK was assessed. The relative sensitivities of the breast cancer cells to the indicated inhibitors, based on their calculated IC50 values, were similar to what has been previously reported in the literature (Additional file 1: Table S1). TNBC cells were comparably sensitive to both $\mathrm{IKK} \varepsilon$ and $\mathrm{IKK} \beta$ inhibition suggesting dependence on NF- $\kappa \mathrm{B}$ classical signaling, whereas the HER2+ cells were more resistant to both inhibitors $(p<0.05$, Fig. 1b-c). HER2-postitive cells were most resistant to IKK $\varepsilon$ inhibition, and sensitivity to classical NF- $\mathrm{kB}$ pathway inhibition was independent of IKK $\varepsilon$ expression level. HER2+ cells were also most resistant to MEK inhibition whereas TNBC cells with the highest IKK $\varepsilon$ expression (MDA MB 468 and MDA MB 231) were most sensitive ( $p<0.05$, Fig. 1d). These data corroborate findings by others showing that IKKe cooperates with MEK to maintain viability.

A western blot was performed on the TNBC cells MDA MB 468 and BT549, which endogenously express high and low $\mathrm{IKK} \varepsilon$, respectively, after 6 -h treatment with the inhibitors. The activity of the IKK $\beta$ and MEK inhibitors was confirmed through western blot analysis of p65 phosphorylation at serine 536 and phosphorylated ERK1/2 (Fig. 2a and Additional file 2: Figure S1a). Interestingly, IKK $\beta$ inhibition reduced phosphorylated ERK1/2 levels only in the presence of $\mathrm{IKK} \varepsilon$, suggesting that MEK activation is, at least partially, regulated by canonical NF- $\mathrm{kB}$ signaling involving IKK $\varepsilon$. We verified the direct effect of IKK $\beta$ inhibition on reduced phosphorylated ERK1/2 at a 30-min time point (Additional file 2: Figure S1b) Inhibition of IKK $\varepsilon$ led to an increase in p100/52, p65, and phosphorylated ERK 1/ 2. Increased protein levels seen with pharmacological IKK $\varepsilon$ inhibition were confirmed using an siRNA against IKBKE $(\operatorname{siIKK\varepsilon })$ in the panel of breast cancer cells that express high endogenous IKKe (Fig. 2b). Indeed, there was an increase in p100/52 and RelB with IKBKE knockdown that approaches significance. This phenomenon was only apparent in the TNBC cells as non-canonical NF-kB signaling proteins are not highly expressed in the HER2+ cells. The MDA 468 cells were then treated with BX795 to inhibit $\mathrm{IKK} \varepsilon$ for $1 \mathrm{~h}$, a shorter time point, and all NF- $\mathrm{kB}$ protein levels were assessed to gain a better understanding of NF- $\mathrm{kB}$ signaling with $\mathrm{IKK} \varepsilon$ inhibition (Fig. 2c). As with the siRNA against IKBKE, the most significantly increased proteins were the non-canonical NF- $\mathrm{\kappa B}$ proteins p100/52 and RelB.

To confirm specificity of IKKe function in TNBC cells IKBKE was knocked down in MDA MB 468 and MDA MB 231 cells and over-expressed in BT-549 (Fig. 3a). Using these complimentary systems, the role of IKK $\varepsilon$ expression and MEK activity was first assessed. As expected, loss of IKK $\varepsilon$ significantly reduced proliferation over 9 days compared to vector control cells. Addition of a MEK inhibitor further reduced viability regardless of IKK $\varepsilon$ expression (Fig. 3b). A similar trend was observed in BT549 cells engineered to express wild-type IKKع. In addition, knockdown of IKBKE resulted in decreased phosphorylation of ERK, and stable over-expression of IKK $\varepsilon$ caused increased ERK phosphorylation (Fig. 3c). Taken together, these results support a model where MEK activation downstream of IKK $\varepsilon$ increases the proliferation of TNBC cells. By western blot, protein levels of the IкB kinases, IKK $\beta$ and IKK $\alpha$, were largely unaffected by $\mathrm{IKK} \varepsilon$, but p52 levels were decreased in the presence of IKK $\varepsilon$ (Fig. 3c). The increased non-canonical NF-kB protein expression with stable $I K B K E$ knockdown was confirmed in the MDA 231 cells and in the MDA 468 cells using an alternate shRNA against IKBKE (Additional file 3: Figure S2).

In order to clarify whether IKKE affected NF-KB transcription factor binding activity, an ELISA immunoassay containing a consensus NF-kB binding sequence was used to evaluate binding activity of downstream NF- $\mathrm{B}$ transcription factors. Binding of the non-canonical transcription factor, p52, was significantly increased when IKKe 

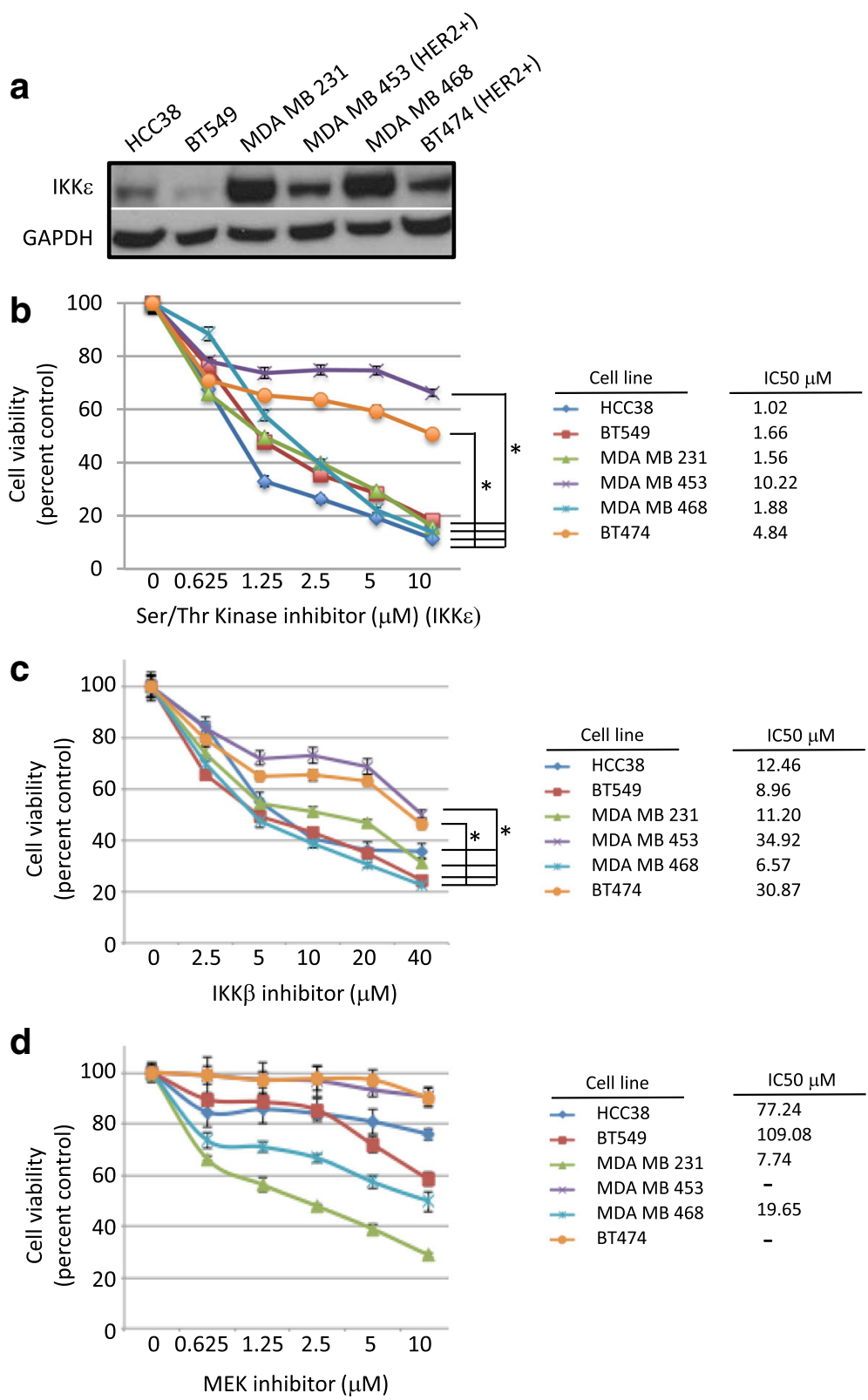

Fig. 1 IKKe expression is variable and correlates with sensitivity to MEK inhibition in TNBC subtype. a) 30 mg of protein was analyzed in whole cell lysates of breast cancer cells grown to $70 \%$ confluence. b-d) 2,000 cells per well were seeded into 96 well plates and allowed to adhere overnight. Inhibitors were added at indicated concentrations and viability assessed via XTT after 72 hours. * IC50 values significantly different between cell lines as indicated, $P<0.05$, one-way ANOVA, comparing each cell line individually. Due to the extreme resistance of cell lines MDA MB 453 and BT474, IC50 values could not be reliably calculated, and statistics are therefore not presented

was knocked down via shRNA relative to a non-targeting control vector (Fig. 4a, left). Similarly, in BT549 lines with endogenously low IKKe expression, binding of p52 was suppressed with exogenous expression of wild-type IKK $\varepsilon$ relative to control vector (Fig. 4a, right). This is consistent with the decreased protein level that was observed on Western blot. On the other hand, IKKe expression did not produce a difference in binding of canonical NF- $\mathrm{kB}$ transcription factors (p65, p50, and C-Rel). These data suggest that while IKKe supports MEK activity, it may interfere with or suppress non-canonical NF- $\kappa B$ activity.

Regulation of NF- $\mathrm{KB}$ target genes CD44 and CXCL1 by p52 in TNBC cells was verified using siRNA knockdown and qRT-PCR. Knockdown of NFKB2 (the gene that encodes p100) led to a small but significant decrease in CXCL1 expression in both lines (Fig. $4 \mathrm{~b}$ and Additional file 4: Figure S3a) indicating transcriptional regulation of CXCL1 by p52. $C D 44$ and $C X C L 1$ have been shown to be regulated by p52 


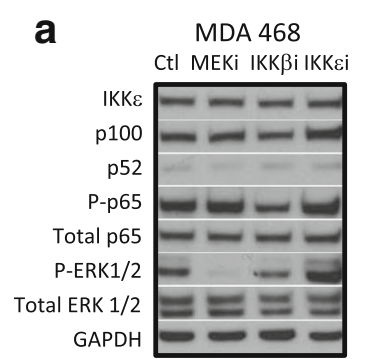

C

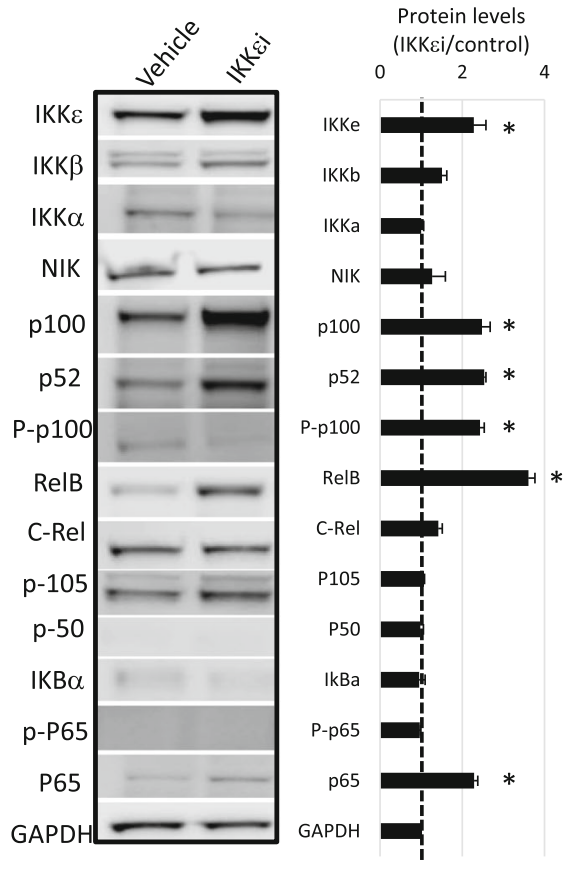

BT549
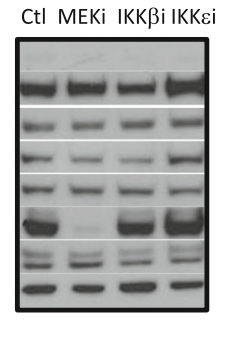

(KKei/control)

P100
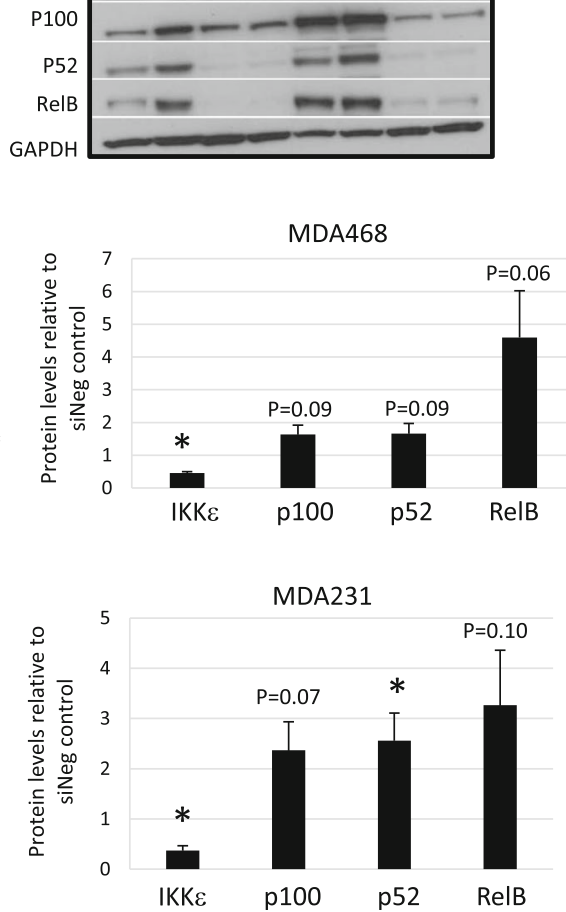

Fig. 2 IKKe supports viability and MEK activation. a) $30 \mu \mathrm{g}$ of protein was analyzed in whole cell lysates of breast cancer cells grown to $70 \%$ confluence. Cells were treated with vehicle control (Ct)), $2 \mu \mathrm{M}$ MEK inhibitor (MEKi), $2 \mu \mathrm{M}$ IKKß inhibitor (IKKßi), or $2 \mu \mathrm{M}$ BX795 for inhibition of IKKE (IKKEi) for $6 \mathrm{~h}$ before lysate collection. b) $30 \mu \mathrm{g}$ of protein was analyzed in whole cell lysates of breast cancer cells grown to $70 \%$ confluence after siRNA mediated knockdown of IKBKE (silKKE). Quantification of three independent replicates reveals increased non-canonical NF-KB proteins with IKBKE knockdown. c) $30 \mu \mathrm{g}$ of protein was analyzed in whole cell lysates of MDA MB 468 cells after $1 \mathrm{~h}$ exposure to $2 \mu \mathrm{M}$ BX795 for IKKE inhibition. Quantification of three independent replicates confirms pharmacological inhibition of IKK $\varepsilon$ activity significantly increased non-canonical NF-KB protein levels. ${ }^{*}$ significantly different from corresponding vehicle control $P<0.05$, unpaired T-test (b-c)

in other systems [30]. Moreover, siRNA knockdown of $I K B K E$, which increases binding activity of p52 (Fig. 4a) led to a significant increase in $R E L B, N F K B 2$ and $C X C L 1$ in the MDA 468 cells (Fig. 4c) suggesting negative transcriptional regulation of alternative NF- $\mathrm{KB}$ transcription factors by IKK $\varepsilon$. In the MDA 231 cells $R E L B, N F K B 2$ and $C D 44$ expression was increased with $I K B K E$ knockdown (Additional file 4: Figure S3b). To confirm that IKK $\varepsilon$ was affecting p52 function, ChIP-PCR was performed to assess the role of IKKe on the binding of $\mathrm{p} 52$ in the CXCL1 promoter. A two-fold enrichment of $\mathrm{p} 52$ binding occurred at a binding site 200 bases upstream of the transcription start site (Fig. 4d). A similar trend was seen in the MDA 231 cells and MDA468 cells transfected with an alternate shRNA against IKBKE (shIKKe
2) (Additional file 4: Figure S3c-d). These data confirm that IKK $\varepsilon$ negatively affects the transcription factor activity of p52. A co-immunoprecipitation assay was performed to determine if IKK $\varepsilon$ is binding to NF-kB inducing Kinase (NIK) or IKK $\alpha$, two upstream kinases responsible for activation of the non-canonical NF- $\kappa B$ pathway (Fig. 4e). Neither NIK nor IKK $\alpha$ co-immunoprecipitated with IKK $\varepsilon$ suggesting IKKe regulates p52 activity through gene transcription and not proteosomal processing of $\mathrm{p} 100$.

The role of p52 and non-canonical NF- $\mathrm{kB}$ activation in breast cancer cells is unclear. Since its expression is inversely correlated with $\mathrm{IKK} \varepsilon$, the non-canonical NF- $\mathrm{kB}$ pathway likely supports a function that is either not dependent on IKKe or repressed by IKKe. Indeed, the 


\section{a}

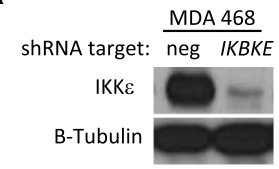

b

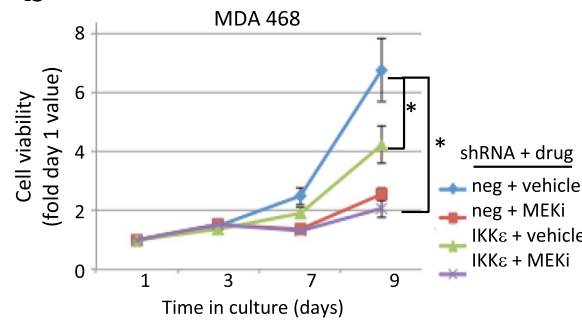

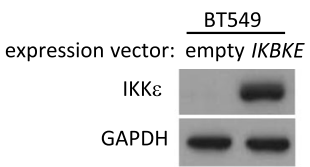

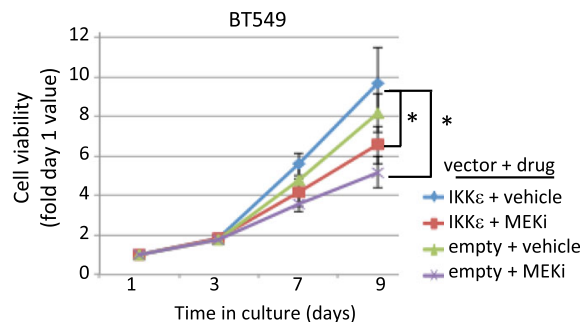

C

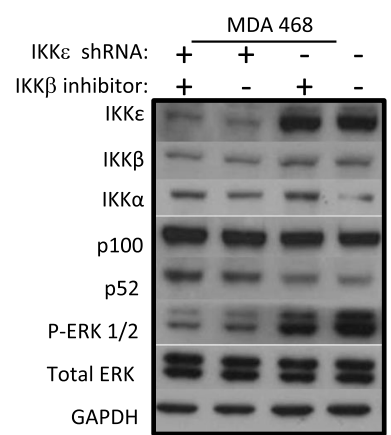

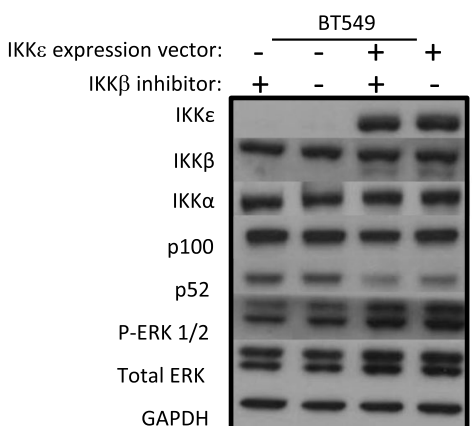

Fig. 3 IKK E expression or activity suppresses non-canonical NF-KB protein expression. a) $50 \mu \mathrm{g}$ of protein was analyzed in whole cell lysates of breast cancer cells engineered to express a constitutively active shRNA against the IKBKE transcript (shIKKE), left, or an expression vector for constitutive synthesis of IKBKE, right. Representative blots show efficiency of shlKKE and expression vector after 8 days selection in puromycin or neomycin, respectively. b) Left, using the MDA-MB-468 cells with stable IKBKE shRNA activity we measured the viability of the cells over a 9 day period. Loss of IKKE significantly impaired survival compared to control. This difference was abrogated in the presence of $1 \mu \mathrm{M}$ MEK inhibitor. Right, the same experiment was performed using the BT549 cells stably transfected with the IKBKE expression vector. ${ }^{*}$ day 9 significantly different from corresponding vehicle control $P<0.05$, one-way ANOVA, post hoc Tukey. c) Left, western blot analysis of MDA MB 468 cells with shRNA-mediated knockdown of IKBKE shows decline in activated MEK (phosphorylated ERK1/2) and increase in p52 levels compared to negative control. Right, stable expression of wild type IKKE is correlated with increased activated MEK and decreased p52. Twenty-four hour exposure $2 \mu \mathrm{M}$ IKK $\beta$ inhibitor had no effect on protein levels

heterogeneity of TNBC cells suggests distinct mechanisms are operating in individual cells to support and maintain phenotypic variability among the population. Therefore, this study interrogated key attributes of cancer cells: proliferation, invasion, resistance to Resistance to anoikis, and spheroid formation. To further explore these aspects, NFKB2 was transiently knocked down using siRNA interference in breast cancer cells expressing high or low levels

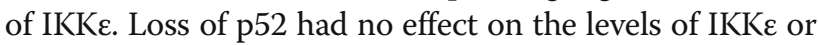
MEK, suggesting their expression or activation are not regulated by non-canonical NF- $\mathrm{kB}$ signaling (Fig. 5a). Furthermore, there was little or no change in viability or invasion potential over $72 \mathrm{~h}$ with loss of p52 (Fig. $5 \mathrm{~b}-\mathrm{c}$ ).

Since IKKE is important for viability of TNBCs, it is important to know whether this phenomenon is also true in low attachment (LA), where cells grow as spheroids after seeding in ultra-low attachment flasks, compared with cells grown as a monolayer in standard high attachment
(HA) culture conditions. Survival and growth in LA conditions implicates resistance to anoikis, apoptosis that occurs in absence of extracellular matrix, and the potential for spheroid growth, both of which are features of carcinogenic cells. Protein levels were assessed by western blot with IKKe and/or p52 knockdown in HA and LA conditions in MDA 468 cells (Fig. 6a). IKK $\varepsilon$ and phosphorylated ERK1/2 protein levels decreased in LA conditions whereas p52 levels increased. These data suggest that while IKKe and MEK support proliferation in both HA and LA conditions, p52 provides a survival advantage specifically in conditions with low anchorage support, indicative of a potential role in spheroid growth. Interestingly, when cultured in LA conditions, knockdown of either IKBKE or $N F K B 2$ alone did not affect relative viability (Fig. $6 \mathrm{~b}$ and Additional file 5: Figure S4a). Knockdown of both IKBKE and $N F K B 2$, however, significantly reduced viability in LA conditions. MEK inhibition decreased viability in both 

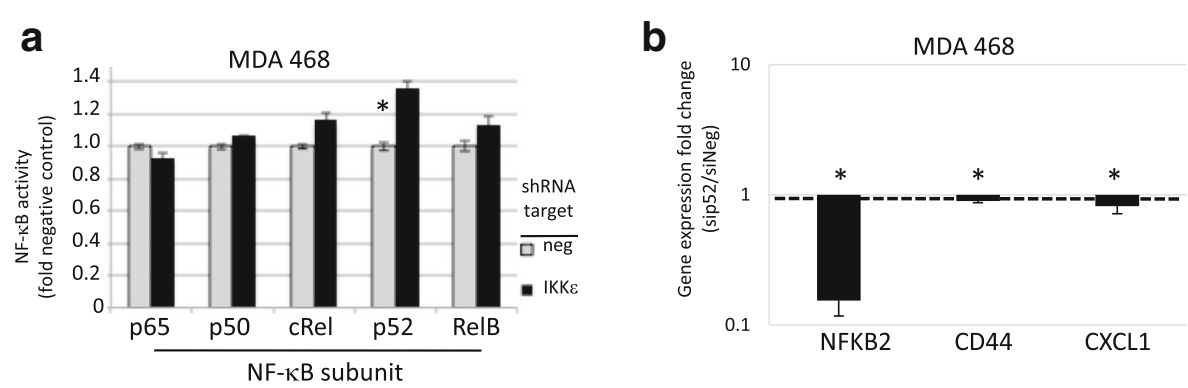

C
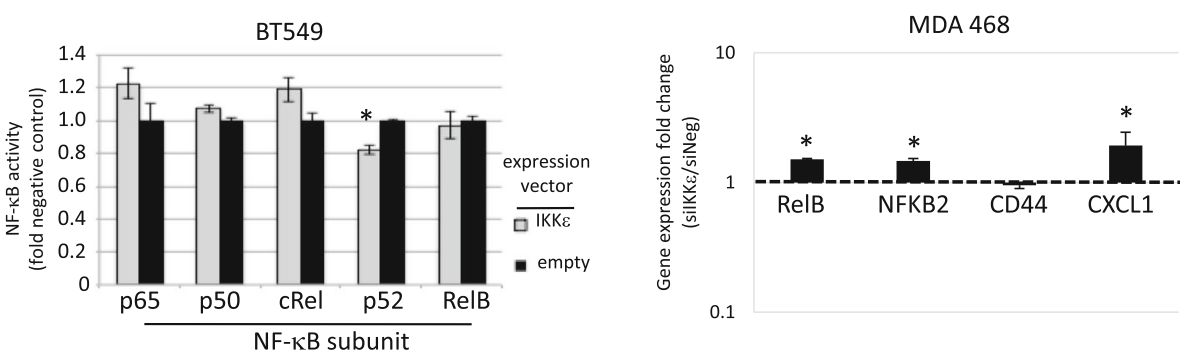

e

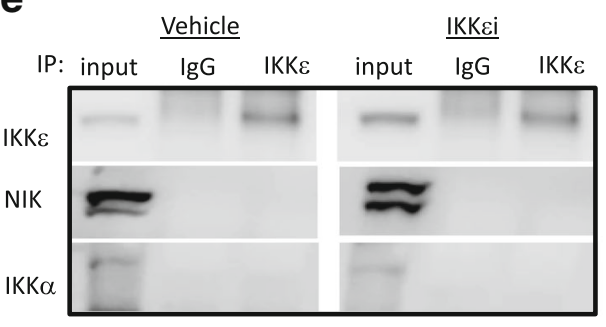

d

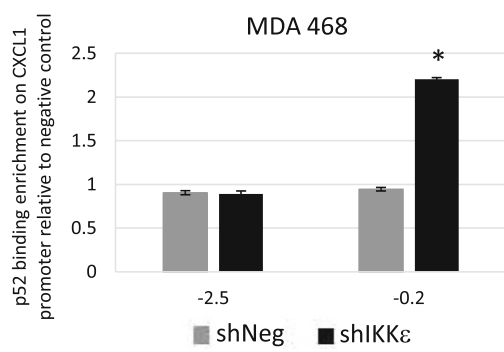

Fig. 4 IKKE inhibits p52 activity independent of interactions with NIK and IKKa. a) Binding activity of the NF-KB p52 transcription factor was significantly decreased in the presence of IKKE. b) siRNA-mediated knockdown of NFKB2 resulted in a significant decrease of CD44 and CXCL1 mRNA expression. c) siRNA-mediated knockdown of IKBKE resulted in a significant increase in mRNA expression of RELB, NFKB2, and CXCL1. d) CHiP-PCR experiments demonstrate significant enrichment of p52 at the promoter of CXCLI when IKKE is knocked down. e) Co-immunoprecipitation experiments indicate IKKE does not interact with NIK or IKKa. ${ }^{*}$ significantly different from corresponding shNeg or siNeg control, $P<0.05$, two-sided unpaired t-test

anchorage-resistant (LA) and anchorage-supportive (HA) plates, and the combination of MEK inhibition and IKBKE knockdown was the most detrimental to cell growth (Fig. $6 \mathrm{c}$ and Additional file 5: Fig. 4b-c). Since p52 supports unattached growth, in vitro spheroid formation was examined. Spheroid formation efficiency was analyzed in the presence and absence of IKK $\varepsilon$ and/or p52 using knockdown of IKBKE and NFKB2, respectively. Once again, loss of either IKK $\varepsilon$ or p52 alone had no effect on spheroid formation efficiency, but loss of both IKKe and p52 significantly reduced spheroid formation potential (Fig. 6d and Additional file 6: Figure S5a-b). Although MEK signaling is important for cell growth and viability, MEK inhibition did not have a significant effect on spheroid formation (Fig. 6e and Additional file 6: Figure S5c).

In summary, this work proposes a model where IKKe and MEK are important for overall proliferative capacity of TNBC cells in all growth conditions, while non-canonical NF- $\mathrm{kB}$ signaling through $\mathrm{p} 52$ is important for survival in anchorage-resistant LA environments. It may be beneficial to the cell to turn off 3D spheroid survival signals when cells are growing in a solid tumor formation attached to extracellular matrix in order to mobilize cellular resources towards replication. Under 3D unattached conditions, such as when metastasizing through the blood stream, both IKK $\varepsilon$ and p52 may be needed to maintain cell viability in suspension at the expense of high proliferation.

\section{Discussion}

NF- $\kappa B$ signaling is important for cancer development, yet the functional role that each subunit plays in this process has not been fully elucidated. IKKe has diverse functions, from activating IRF3 and IRF7 as part of an anti-viral response [31]. This kinase has also been shown to activate canonical NF-kB signaling by directly phosphorylating the subunit RelA/p65 [32]. IKKe is an oncogene in breast cancer with constitutive expression in some breast cancer cell lines and patient samples. Several studies, including this 
a

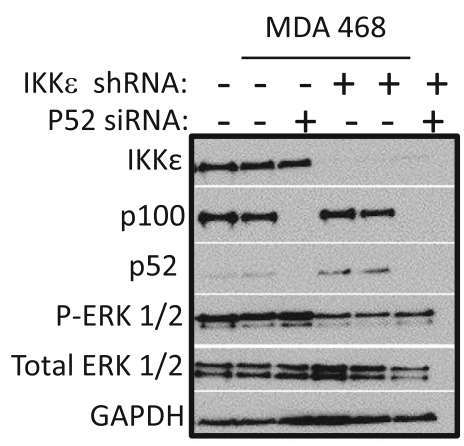

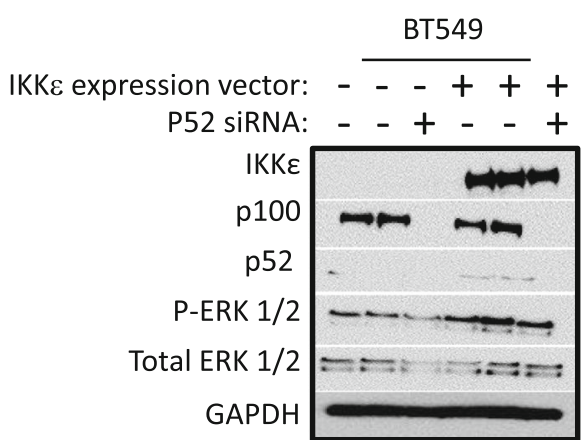

GAPDH

ns

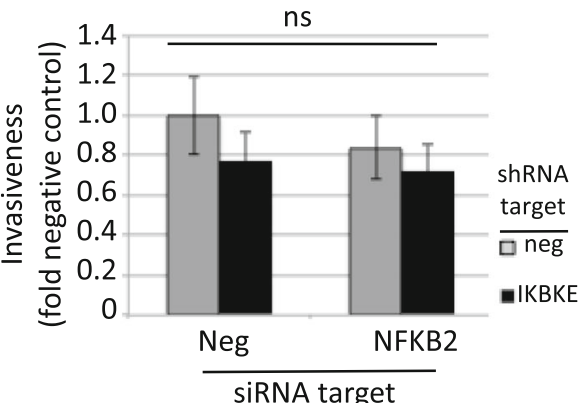

BT549

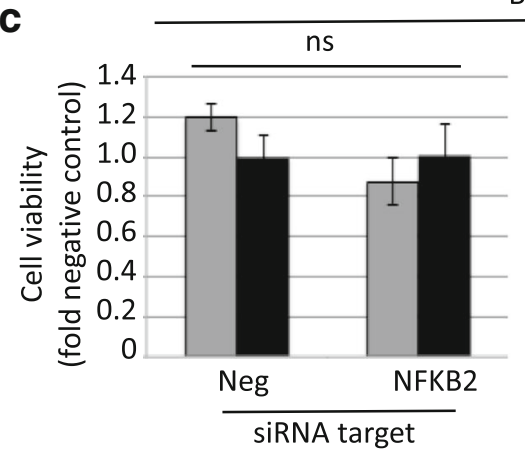

Fig. 5 p52 does not contribute to viability or invasive potential. a) Using the MDA-MB-468 cells with stably knocked down IKBKE, we transiently knocked down NFKB2 using siRNA interference. Representative western blots shown using $30 \mu \mathrm{g}$ protein lysate collected $72 \mathrm{~h}$ following siRNA transfection. b) Cell viability over $72 \mathrm{~h}$ was not significantly altered by loss of IKKE or p52, left. Similarly, loss of IKKE or p52 had no effect on invasiveness at $72 \mathrm{~h}$, right. c) Viability, left, and invasion, right, experiments were repeated using the BT549 cells stably expressing IKKE. ns, not significant according to one-way ANOVA, post hoc-Tukey

one, shed light on the functional significance of this protein in breast cancer progression [33,34]. The role of IKKe is of particular importance given the lack of therapeutic targets in TNBC and the deficient characterization of this protein.

The kinase activity of IKKe positively regulates the promoter regions of cyclin D1 and RelB in TNBC, and loss of IKKE activity diminished the cells ability to grow in soft agar and form colonies in Matrigel [33]. In another setting, IKK controlled constitutive phosphorylation of p65 to positively regulate proliferation of HeLa cells [34]. Also in HeLa cells, IKK $\varepsilon$ directly interacts with a complex containing both p65 and $\mathrm{p} 52$, and phosphorylation of $\mathrm{p} 65$ by IKK $\varepsilon$ resulted in the transactivation of p52 [35]. In contrast to HeLa cells, our data show that IKK $\varepsilon$ increases MEK activation and decreases p52 activity in TNBC. This study further shows that protein levels of p52 increase while activated MEK decreases in LA conditions, regardless of IKK $\varepsilon$ expression. These data suggest that IKKe supports long-term viability in diverse environments but that p52 is required to maintain this property in anchorage resistant LA cultures, where cell death occurs within days. Non-canonical NF-kB signaling assists in the development of breast cancer spheroids and may play a larger role in 3-D growth dynamics compared with MEK signaling. This study expands on recent reports by our laboratory and 


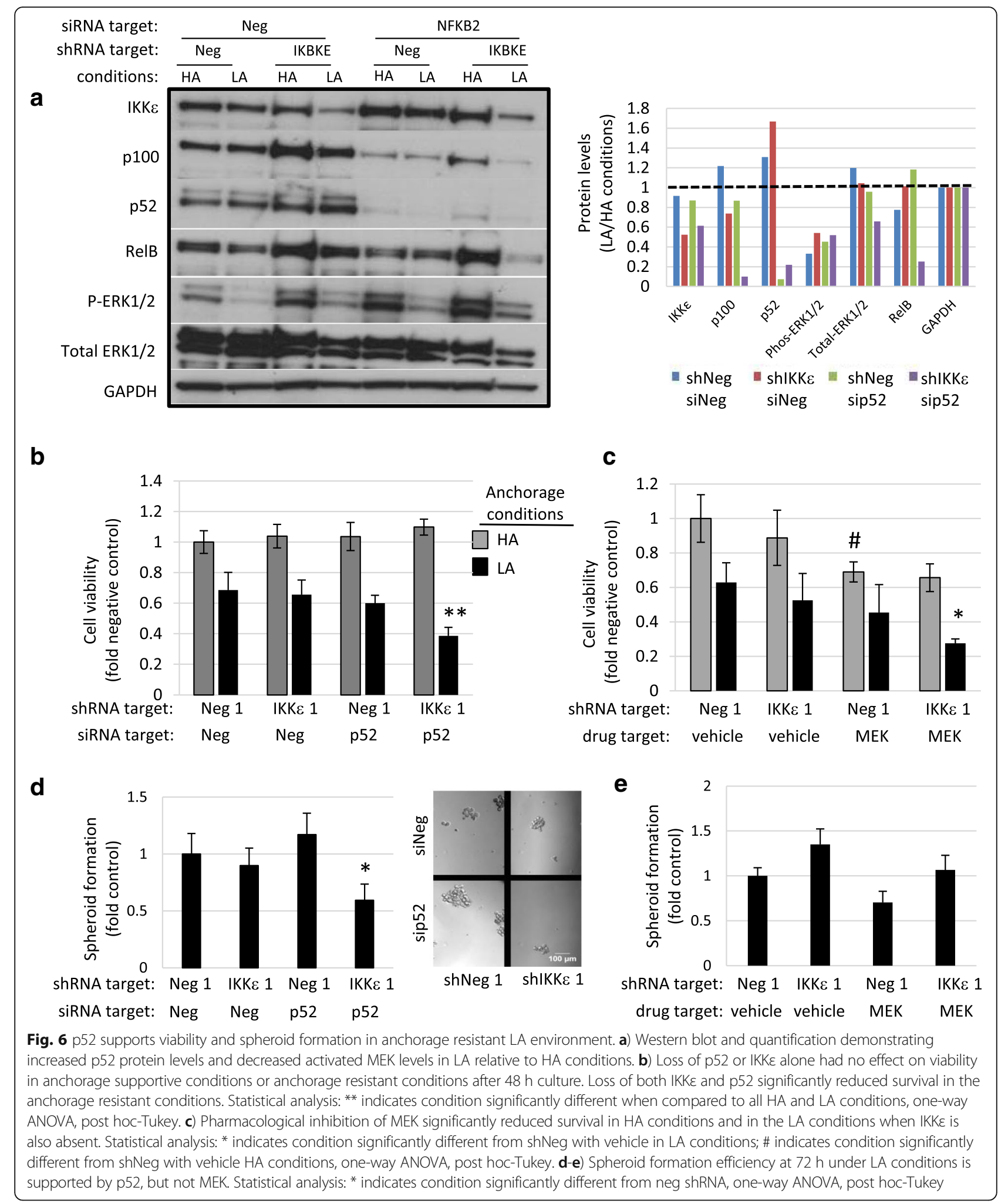

others highlighting a role for non-canonical NF- $\mathrm{kB}$ signaling supporting a cancer spheroid phenotype [17, 36-38].

Together, these studies further illustrate the complex crosstalk associated with NF-kB signaling in maintaining cancer cell survival, and highlight the varied roles of IKKe. This diversity is especially critical in the context of cancer where plasticity is advantageous for cell survival. Although $\mathrm{IKK} \varepsilon$ expression is inversely proportional to $\mathrm{p} 52$, both are 
required for growth in LA. This suggests that, while some of their functions may overlap in HA conditions, these two proteins may also serve independent roles in sustaining the LA cells. Current literature states that canonical and non-canonical substrates can be targeted by IKK $\varepsilon$ to induce NF- $\mathrm{kB}$ activation [39]. Importantly, both canonical and non-canonical NF- $\mathrm{kB}$ signals support cancer cell progression and the crosstalk with other signaling pathways provides additional mechanisms for oncogenesis [21, 37]. This may be especially important in a changing tumor cell microenvironment. For example, in the reversible process of epithelial-to-mesenchymal transition, cellular requirements differ as the cell fluxes between a solid primary tumor, shedding into the lympho-vascular system, and re-settling into a micrometastatic niche. Breast cancer stem cells are known to have this capacity to transition between epithelial and mesenchymal states, and it has been shown that NF- $\mathrm{kB}$ can regulate this process $[37,40]$. The complex interaction between $\mathrm{IKK} \varepsilon$ and $\mathrm{p} 52$ requires further study in order to identify the optimal point of intervention when designing therapeutics to target these pathways.

In light of these findings and our current study, it is plausible that IKKe supports proliferation via canonical MEK activation in anchorage supportive conditions and viability via non-canonical NF-kB p52 signaling in anchorage-resistant conditions. These findings underscore the key role of IKK $\varepsilon$ in both primary tumors and the metastatic process. Importantly, our previous work showed that IKKe was higher in metastatic ovarian cancer, and was crucial to the metastatic process in a mouse model [24]. The results presented herein underscore a similar function for IKKe in TNBC, and designate a cooperative role for non-canonical NF- $\mathrm{kB}$ signaling in resisting anoikis and forming spheroids.

\section{Conclusions}

There is dire need to develop better combinatorial therapeutics for combating TNBC. IKK $\varepsilon$ is a diverse kinase capable of supporting viability of TNBC cells under different cellular contexts. Our study suggests that IKKe cooperates with MEK to support proliferation whereas IKK $\varepsilon$ directs cellular resources to $\mathrm{p} 52$ to support viability in an anchorage resistant or mobile setting. A better understanding of the function of IKK $\varepsilon$ within the NF-kB signaling network as well as with other major signaling pathways will likely provide alternative therapeutic strategies for patients with TNBC.

\section{Additional files}

Additional file 1: Table S1. IC50 values for selected inhibitors in breast cancer lines. IC50 values obtained in the current study listed with IC50 values in same cell lines obtained from public database www.cancerrxgene.org. (PPTX $40 \mathrm{~kb}$ )

Additional file 2: Figure S1. Protein activity suppressed by inhibitors. Western blot quantification and short time point for phospho-ERK western blot. a) Quantification of western blot represented in Figure 2a showing protein level changes in BT549 or MDA MB 468 cells after $6 \mathrm{~h}$ treatment with indicated inhibitors. Quantification is relative to vehicle control lanes. b) Western blot showing decrease in phosphorylated ERK1/2 upon 30-min exposure to IKKE inhibitor, and accompanying graph for quantitation of phospho-ERK, as normalized to GAPDH, and relative to expression in untreated MDA468. (PPTX $429 \mathrm{~kb}$ )

Additional file 3: Figure S2. Knockdown of IKKE leads to increased expression of non-canonical NF-kB proteins in at least two TNBC lines. Western blot and quantification of additional shRNA and data in MDA MB 231 cell line. An alternate shRNA sequence against IKBKE was expressed in MDA MB 468 cells and in MDA MB 231 cells to show specificity and an additional TNBC model. (PPTX $200 \mathrm{~kb}$ )

Additional file 4: Figure S3. IKKE inhibits activity of p52. qRT-PCR and ChIP-PCR results for MDA MB 231 cell line and additional shRNA in MDA MB 468 cell line. a) siRNA-mediated knockdown of NFKB2 in MDA MB 231 cells led to a significant decrease in CXCL1 expression. b) siRNAmediated knockdown of IKBKE in MDA MB 231 cells increased expression of RELB, NFKB2, and CD44. c) Loss of IKKE in MDA MB 231 cells led to a significant enrichment of p52 binding on the promoter of the CXCL1 gene. d) Similar results were seen in MDA MB 468 cells expressing an alternate shRNA against IKBKE (shIKKع 2). (PPTX 64 kb)

Additional file 5: Figure S4. IKKE and $\mathrm{p} 52$ or MEK supports viability in $L A$ conditions in at least two TNBC lines. Growth conditions and anoikis data with additional shRNA in MDA MB 468 cell line and in MDA MB 231 cell line. a) Left, expressing an alternate shRNA for IKBKE in MDA MB 468 cells supports the data shown in Fig. 6b. Right, similar trends were also seen in the MDA MB 231 line. b) MEK inhibition in presence of alternate shRNA against IKBKE led to similar outcomes as shown in Figure $6 c$. Viability of MDA MB 231 cells is more dependent on MEK signaling than IKKE. c) Western blot verifying IKK $\varepsilon$ and p52 knockdown in MDA MB 231 cells. Statistical analysis: ${ }^{*}$ indicates condition significantly different as indicated by bars; ${ }^{* *}$ indicates condition significantly different when compared to all HA and LA conditions, one-way ANOVA, post hoc-Tukey. (PPTX 392 kb)

Additional file 6: Figure S5. IKKE and p52 or MEK supports viability in LA conditions in at least two TNBC lines. Spheroid formation data with additional shRNA in MDA MB 468 cell line and in MDA MB 231 cell line. a) An alternate shRNA for IKBKE in MDA MB 468 cells supports the data shown in Figure $6 \mathrm{~d}$ that p52 and IKKE are both necessary for efficient spheroid formation. b) The MDA MB 231 was more dependent on p52 for efficient spheroid formation as knockdown of IKKE had no effect or slightly enhanced spheroid formation c) MEK had no effect on spheroid formation in MDA MB 231 cells however knockdown of IKKe enhanced spheroid formation efficiency. (PPTX $368 \mathrm{~kb}$ )

\section{Abbreviations}

BME: Basement membrane extract; EGF: Epidermal growth factor; ERK: Extracellular signal-related kinase; FBS: Fetal bovine serum; FGF: Fibroblast growth factor; HA: High attachment culture conditions; HER2: Human epidermal growth factor receptor 2; IKKa: Inhibitor of nuclear factor kappa-B kinase subunit alpha; IKKß: Inhibitor of nuclear factor kappa-B kinase subunit beta; IKKE: Inhibitor of nuclear factor kappa-B kinase subunit epsilon; LA: Low attachment culture conditions; MEK: Mitogen-activated protein/extracellular signal-regulated kinase kinase; NF-KB: Nuclear factor kappa-light-chain-enhancer of activated B cells; ns: Not significant; RPMI: Roswell Park Memorial Institute; shRNA: Short hairpin RNA; siRNA: Short interfering RNA; TNBC: Triple-negative breast cancer; XTT: Tetrazolium dye

\section{Acknowledgements}

The authors wish to thank Dr. Patricia Steeg and Dr. Stanley Lipkowitz for helpful discussions. CDH was supported by the National Institute for General Medical Sciences Postdoctoral Research Associate Training (PRAT) Program.

\section{Funding}

Financial support provided by Intramural Research Program (ZIA BC 011054), National Cancer Institute, Center for Cancer Research, Bethesda, Maryland. The funding agency had no role in study design, data analysis, interpretation of results, or in the writing of the manuscript. 


\section{Availability of data and materials}

The datasets used and/or analyzed during the current study are available from the corresponding author on reasonable request.

\begin{abstract}
Authors' contributions
$\mathrm{CDH}$ and VG designed experiments and executed viability assays, western blots, invasion studies, binding assays, siRNA transfections, ChIP-qRT-PCR, Coimmunoprecipitations. $\mathrm{MO}$ and EJ performed western blots, siRNA transfections, spheroid formation assays. HW and DCK helped conceive and design the project, created stably transfected cell lines, and performed western blots. JMJ and $\mathrm{CDH}$ performed siRNA transfections and anoikis assays. MKK designed and coordinated experiments and helped to draft the manuscript. CMA conceived and designed the study. $\mathrm{CDH}$ and CMA performed statistical analysis and wrote the manuscript. All authors read and approved the final manuscript.
\end{abstract}

\section{Ethics approval and consent to participate}

Not applicable.

\section{Competing interests}

The authors declare that they have no competing interests.

\section{Publisher's Note}

Springer Nature remains neutral with regard to jurisdictional claims in published maps and institutional affiliations.

Received: 13 February 2018 Accepted: 15 May 2018

Published online: 25 May 2018

\section{References}

1. Siegel R, Naishadham D, Jemal A. Cancer statistics, 2013. CA Cancer J Clin. 2013;63(1):11-30. https://doi.org/10.3322/caac.21166.

2. Lehmann BD, Bauer JA, Chen X, Sanders ME, Chakravarthy AB, Shyr Y, et al. Identification of human triple-negative breast cancer subtypes and preclinical models for selection of targeted therapies. J Clin Invest. 2011; 121(7):2750-67. https://doi.org/10.1172/JCl45014

3. Prat A, Adamo B, Cheang MC, Anders CK, Carey LA, Perou CM. Molecular characterization of basal-like and non-basal-like triple-negative breast cancer. Oncologist. 2013;18(2):123-33. https://doi.org/10.1634/theoncologist. 2012-0397.

4. Mayer IA, Abramson VG, Lehmann BD, Pietenpol JA. New strategies for triple-negative breast cancer-deciphering the heterogeneity. Clin Cancer Res. 2014;20(4):782-90. https://doi.org/10.1158/1078-0432.CCR-13-0583.

5. Ossovskaya V, Wang Y, Budoff A, Xu Q, Lituev A, Potapova O, et al. Exploring molecular pathways of triple-negative breast cancer. Genes Cancer. 2011; 2(9):870-9. https://doi.org/10.1177/1947601911432496.

6. Bendinelli P, Matteucci E, Maroni P, Desiderio MA. NF-kappaB activation, dependent on acetylation/deacetylation, contributes to HIF-1 activity and migration of bone metastatic breast carcinoma cells. Mol Cancer Res. 2009; 7(8):1328-41. https://doi.org/10.1158/1541-7786.MCR-08-0548.

7. Hoeflich KP, O'Brien C, Boyd Z, Cavet G, Guerrero S, Jung K, et al. In vivo antitumor activity of MEK and phosphatidylinositol 3-kinase inhibitors in basal-like breast cancer models. Clin Cancer Res. 2009;15(14):4649-64. https://doi.org/10.1158/1078-0432.CCR-09-0317.

8. Ibrahim YH, García-García C, Serra V, He L, Torres-Lockhart K, Prat A, et al. PI3K inhibition impairs BRCA1/2 expression and sensitizes BRCA-proficient triple-negative breast cancer to PARP inhibition. Cancer Discov. 2012;2(11): 1036-47. https://doi.org/10.1158/2159-8290.CD-11-0348.

9. Kim HS, Yom CK, Kim HJ, Lee JW, Sohn JH, Kim JH, et al. Overexpression of p53 is correlated with poor outcome in premenopausal women with breast cancer treated with tamoxifen after chemotherapy. Breast Cancer Res Treat. 2010;121(3):777-88. https://doi.org/10.1007/s10549-009-0560-5.

10. Stemke-Hale K, Gonzalez-Angulo AM, Lluch A, Neve RM, Kuo WL, Davies M, et al. An integrative genomic and proteomic analysis of PIK3CA, PTEN, and AKT mutations in breast cancer. Cancer Res. 2008;68(15):6084-91. https:// doi.org/10.1158/0008-5472.CAN-07-6854.

11. Sen R, Baltimore D. Inducibility of kappa immunoglobulin enhancer-binding protein Nf-kappa B by a posttranslational mechanism. Cell. 1986;47(6):921-8.

12. Duckett CS. Apoptosis and NF-kappa B: the FADD connection. J Clin Invest. 2002;109(5):579-80. https://doi.org/10.1172/JCl15197.
13. Guttridge DC, Albanese C, Reuther JY, Pestell RG, Baldwin AS. NF-kappaB controls cell growth and differentiation through transcriptional regulation of cyclin D1. Mol Cell Biol. 1999;19(8):5785-99.

14. La Rosa FA, Pierce JW, Sonenshein GE. Differential regulation of the c-myc oncogene promoter by the NF-kappa B rel family of transcription factors. Mol Cell Biol. 1994;14(2):1039-44.

15. Pikarsky E, Porat RM, Stein I, Abramovitch R, Amit S, Kasem S, et al. NF-kappaB functions as a tumour promoter in inflammation-associated cancer. Nature. 2004;431(7007):461-6. https://doi.org/10.1038/nature02924.

16. Hayden MS, Ghosh S. Signaling to NF-kappaB. Genes Dev. 2004;18(18):2195-224. https://doi.org/10.1101/gad.1228704.

17. House CD, Jordan E, Hernandez L, Ozaki M, James JM, Kim M, et al. NFKB promotes ovarian tumorigenesis via classical pathways that support proliferative Cancer cells and alternative pathways that support ALDH+ Cancer stem-like cells. Cancer Res. 2017;77(24):6927-40. https://doi.org/10. 1158/0008-5472.CAN-17-0366.

18. Oeckinghaus A, Hayden MS, Ghosh S. Crosstalk in NF-KB signaling pathways. Nat Immunol. 2011;12(8):695-708. https://doi.org/10.1038/ni.2065.

19. Kucharczak J, Simmons MJ, Fan Y, Gélinas C. To be, or not to be: NF-kappaB is the answer-role of Rel/NF-kappaB in the regulation of apoptosis. Oncogene. 2003:22(56):8961-82. https://doi.org/10.1038/sj.onc.1207230.

20. Boehm JS, Zhao JJ, Yao J, Kim SY, Firestein R, Dunn IF, et al. Integrative genomic approaches identify IKBKE as a breast cancer oncogene. Cell. 2007; 129(6):1065-79. https://doi.org/10.1016/j.cell.2007.03.052.

21. Perkins ND. The diverse and complex roles of NF-kB subunits in cancer. Nat Rev Cancer. 2012;12(2):121-32. https://doi.org/10.1038/nrc3204.

22. Barham W, Chen L, Tikhomirov O, Onishko H, Gleaves L, Stricker TP, et al. Aberrant activation of NF-kB signaling in mammary epithelium leads to abnormal growth and ductal carcinoma in situ. BMC Cancer. 2015;15:647. https://doi.org/10.1186/s12885-015-1652-8.

23. Annunziata CM, Stavnes HT, Kleinberg L, Berner A, Hernandez LF, Birrer MJ et al. Nuclear factor kappaB transcription factors are coexpressed and convey a poor outcome in ovarian cancer. Cancer. 2010;116(13):3276-84. https://doi.org/10.1002/cncr.25190.

24. Hsu S, Kim M, Hernandez L, Grajales V, Noonan A, Anver M, et al. IKK- $\varepsilon$ coordinates invasion and metastasis of ovarian cancer. Cancer Res. 2012 72(21):5494-504. https://doi.org/10.1158/0008-5472.CAN-11-3993.

25. Hutti JE, Shen RR, Abbott DW, Zhou AY, Sprott KM, Asara JM, et al. Phosphorylation of the tumor suppressor CYLD by the breast cancer oncogene IKKepsilon promotes cell transformation. Mol Cell. 2009;34(4):46172. https://doi.org/10.1016/j.molcel.2009.04.031.

26. Barbie TU, Alexe G, Aref AR, Li S, Zhu Z, Zhang X, et al. Targeting an IKBKE cytokine network impairs triple-negative breast cancer growth. J Clin Invest. 2014;124(12):5411-23. https://doi.org/10.1172/JCl75661.

27. Qin B, Cheng K. Silencing of the IKKE gene by siRNA inhibits invasiveness and growth of breast cancer cells. Breast Cancer Res. 2010;12(5):R74. https:// doi.org/10.1186/bcr2644

28. Scudiero DA, Shoemaker RH, Paull KD, Monks A, Tierney S, Nofziger TH, et al. Evaluation of a soluble tetrazolium/formazan assay for cell growth and drug sensitivity in culture using human and other tumor cell lines. Cancer Res. 1988:48(17):4827-33.

29. Schneider CA, Rasband WS, Eliceiri KW. NIH image to ImageJ: 25 years of image analysis. Nat Methods. 2012;9(7):671-5.

30. Nadiminty N, Dutt S, Tepper C, Gao AC. Microarray analysis reveals potential target genes of NF-kappaB2/p52 in LNCaP prostate cancer cells. Prostate. 2010;70(3):276-87. https://doi.org/10.1002/pros.21062.

31. Nakatsu Y, Matsuoka M, Chang TH, Otsuki N, Noda M, Kimura H, et al. Functionally distinct effects of the C-terminal regions of IKKE and TBK1 on type I IFN production. PLoS One. 2014;9(4):e94999. https://doi.org/10.1371/ journal.pone.0094999.

32. Wang $Y$, Lu $X$, Zhu L, Shen $Y$, Chengedza S, Feng $H$, et al. IKK epsilon kinase is crucial for viral G protein-coupled receptor tumorigenesis. Proc Natl Acad Sci U S A. 2013;110(27):11139-44. https://doi.org/10.1073/pnas.1219829110.

33. Eddy SF, Guo S, Demicco EG, Romieu-Mourez R, Landesman-Bollag E, Seldin DC, et al. Inducible IkappaB kinase/lkappaB kinase epsilon expression is induced by CK2 and promotes aberrant nuclear factor-kappaB activation in breast cancer cells. Cancer Res. 2005;65(24):11375-83. https://doi.org/10. 1158/0008-5472.CAN-05-1602.

34. Adli M, Baldwin AS. IKK-i//KKepsilon controls constitutive, cancer cell-associated NF-kappaB activity via regulation of Ser-536 p65/RelA phosphorylation. J Biol Chem. 2006;281(37):26976-84. https://doi.org/10.1074/jbc.M603133200. 
35. Wietek C, Cleaver CS, Ludbrook V, Wilde J, White J, Bell DJ, et al. IkappaB kinase epsilon interacts with p52 and promotes transactivation via p65. Biol Chem. 2006;281(46):34973-81. https://doi.org/10.1074/jbc.M607018200.

36. Yeo SK, French R, Spada F, Clarkson R. Opposing roles of Nfkb2 gene products p100 and p52 in the regulation of breast cancer stem cells. Breast Cancer Res Treat. 2017;162(3):465-77. https://doi.org/10.1007/s10549-017-4149-0.

37. Kendellen MF, Bradford JW, Lawrence CL, Clark KS, Baldwin AS. Canonical and non-canonical NF-KB signaling promotes breast cancer tumor-initiating cells. Oncogene. 2014;33(10):1297-305. https://doi.org/10.1038/onc.2013.64.

38. Rojo F, González-Pérez A, Furriol J, Nicolau MJ, Ferrer J, Burgués O, et al. Non-canonical NF-kB pathway activation predicts outcome in borderline oestrogen receptor positive breast carcinoma. Br J Cancer. 2016;115(3):322-31. https://oi.org/10.1038/bjc.2016.204.

39. Shen RR, Hahn WC. Emerging roles for the non-canonical IKKs in cancer. Oncogene. 2011;30(6):631-41. https://doi.org/10.1038/onc.2010.493.

40. Liu S, Cong Y, Wang D, Sun Y, Deng L, Liu Y, et al. Breast Cancer stem cells transition between epithelial and mesenchymal states reflective of their normal counterparts. Stem Cell Reports. 2014;2(1):78-91. https://doi.org/10 1016/..stemcr.2013.11.009.

Ready to submit your research? Choose BMC and benefit from:

- fast, convenient online submission

- thorough peer review by experienced researchers in your field

- rapid publication on acceptance

- support for research data, including large and complex data types

- gold Open Access which fosters wider collaboration and increased citations

- maximum visibility for your research: over $100 \mathrm{M}$ website views per year

Learn more biomedcentral.com/submissions 